\title{
Avaliação do risco coronariano em idosos portadores de hipertensão arterial em tratamento
}

\author{
Evaluation of coronary risk for hypertensive elderly under treatment
}

Célida Juliana de Oliveira', Maria Josefina da Silva', Paulo César de Almeida², Thereza Maria Magalhães Moreira²

\begin{abstract}
Resumo
Diversos estudos revelam uma grande proximidade dos fatores para o desenvolvimento e/ou agravamento da hipertensão arterial e os fatores de risco para a doença coronariana e suas complicações. Objetivamos averiguar o risco coronariano, ao qual idosos em tratamento anti-hipertensivo estão submetidos, com vistas a subsidiar o cuidado de enfermagem a esse grupo. Estudo descritivo transversal quantitativo, aprovado pelo Comitê de Ética em Pesquisa da Universidade Estadual do Ceará e realizado junto a 54 idosos, de ambos os sexos, em tratamento farmacológico para hipertensão arterial. A coleta de dados ocorreu entre agosto e setembro de 2007, por meio de um formulário de identificação e aplicação da tabela de risco coronariano. As características prevalentes foram: sexo feminino $(74,1 \%)$, faixa etária entre 70 e 79 anos $(48,1 \%)$, idosos casados $(50 \%)$, escolaridade inferior a oito anos de estudos $(46,3 \%)$, renda mensal de um salário mínimo $(51,9 \%)$. Valores não controlados da pressão arterial foram registrados entre $22 \%$ e $25 \%$ dos idosos e o risco coronariano prevalente foi o moderado (55\%). Concluiu-se que, apesar dos idosos terem se mostrado com estado de saúde cardiovascular regular, muito pode ser feito pela enfermagem para ampliar seu cuidado. 0 enfermeiro deve elaborar estratégias para intervir nos fatores de risco modificáveis, do desenvolvimento de doenças cardio-cerebro-vasculares visando reduzir a morbi-mortalidade dos idosos.
\end{abstract}

Palavras-chave: Idosos; hipertensão; enfermagem; doença das coronárias.

\section{Abstract}

Several studies reveal a great proximity of the factors for the development and deterioration of the arterial hypertension and the risk factors for the coronary disease and its complications. We aimed to investigate the coronary risk to elderly in antihypertensive treatment, providing information for the nursing care to that group. This descriptive, transversal and quantitative study was approved for the Committee of Ethics in Research of the Universidade Estadual do Ceará and accomplished with 54 elderlies, of both sexes, in pharmacological treatment for arterial hypertension. Data was collected happened between august and september of 2007 through an identification form and application of the table of coronary risk. Prevalent characteristics were: female sex (74.1\%), age group between 70 and 79 years $(48.1 \%)$, married elderly $(50 \%)$, education up to eight years of studies (46.3\%), familial earnings under minimum wage (51.9\%). Values not controlled of the blood pressure were registered between $22 \%$ and $25 \%$ of the elderly and the prevalent coronary risk was the moderate (55\%). We concluded that, in spite of the elderly to be shown with regular cardiovascular health condition, much can be done by the nursing to improve care. The nurses should elaborate strategies to intervene in modifiable risk factors of development of cardiobrain-vascular diseases, reducing the elderly morbi-mortality.

Keywords: Elderly; hypertension; nursing; coronary disease

Recebido: 03/09/2008

Revisado: 11/10/2008

Aprovado: $16 / 10 / 2008$

Universidade Federal do Ceará (UFC), Fortaleza, CE

2 Universidade Estadual do Ceará (UECE), Fortaleza, CE

Pesquisa derivada da dissertação de CJO, defendida no Programa de Mestrado Acadêmico Cuidados Clínicos em Saúde da Universidade Estadual do

Ceará (UECE) e financiada por bolsa da Fundação Cearense de Apoio ao Desenvolvimento Científico e Tecnológico (FUNCAP)

Endereço para correspondência: Célida Juliana de Oliveira/Rua dos Jatobás, 373, casa 25 - Passaré, CEP: $60743-840$ - Fortaleza (CE),

E-mail: celidajuliana@yahoo.com.br 


\section{Introdução}

O envelhecimento humano é um processo gradual, irreversível e incontrolável de declínio das funções fisiológicas ${ }^{1}$, sendo um evento cada vez mais comum nos dias atuais, na maioria dos países do mundo, inclusive no Brasil.

A promoção do envelhecimento saudável é uma tarefa multidisciplinar, e a enfermagem tem um papel relevante, como um dos promotores do envelhecimento ativo e saudável, em que o indivíduo possa aproveitar oportunidades que favoreçam a manutenção do seu bem-estar ao longo da vida ${ }^{2}$, visando mais especificamente, a redução da morbidade física, psicológica e social do idoso que está mais propício aos efeitos e consequiências de diversos distúrbios, especialmente as doenças crônicas.

Dentre as doenças crônico-degenerativas mais prevalentes nesta faixa etária, destaca-se a hipertensão arterial (HA), que, por ser uma entidade multifatorial e depender da colaboração e participação ativa do indivíduo hipertenso para seu controle, constitui-se em um grande desafio para os profissionais de saúde ${ }^{3}$.

Se não tratada adequadamente, a HA pode acarretar graves conseqüências para o indivíduo, estando entre as causas mais freqüentes de morbi-mortalidade dos adultos e idosos. A doença é responsável por $25 \%$ das mortes por doença arterial coronariana no Brasil ${ }^{4}$, sendo imperativa a reflexão dos profissionais da saúde sobre a gravidade das conseqüências desses eventos, para que os mesmos possam orientar e estimular o portador de hipertensão a modificar hábitos nocivos à sua saúde, auxiliando-o a controlar seus níveis pressóricos.

Diversos estudos revelam uma grande proximidade dos fatores para o desenvolvimento/agravamento da hipertensão arterial e os fatores de risco para a doença coronariana e suas complicações, já que é sabido que, dentre os fatores de risco alteráveis para eventos coronarianos, a hipertensão arterial é forte contribuinte.

Stuchi e Carvalho ${ }^{5}$ ressaltam uma forma de classificação dos fatores de risco associados ao desenvolvimento de doenças coronarianas: inalteráveis, que são a idade, o sexo, a raça e a presença de antecedentes familiares e alteráveis, que são a própria HA, dislipidemias, o tabagismo, diabetes, obesidade, vida sedentária, uso de anticoncepcionais hormonais.

Um estudo brasileiro apresentado em 1997, observou prevalência geral de fatores de risco de $93 \%$, sendo que a hipertensão apresentou índice de 53\%, entre idosos de 65 a 95 anos, o que reforça a idéia de que estando com os níveis pressóricos controlados, o indivíduo terá um índice de risco menor para o surgimento ou agravamento de seu quadro cardiovascular ${ }^{6}$

A atuação da enfermagem sobre os fatores de risco passíveis de modificação (os ambientais, de estilo de vida ou alteráveis) é de extrema importância para evitar o desenvolvimento da hipertensão arterial e/ou suas complicações, entre elas, as doenças coronarianas ${ }^{7}$. As alterações executadas nesse nível, na maioria das vezes, são suficientes para mini- mizar os efeitos dos fatores de risco constitucionais. Vale destacar que, apesar da hipertensão primária não ter cura, sua detecção, intervenção nos fatores de risco e tratamento precoces possibilitam seu controle e reduzem complicações.

Ao conhecer quais variáveis interferem ou auxiliam no tratamento anti-hipertensivo, o enfermeiro tem dados concretos para ajudar o idoso a dar um correto seguimento à terapêutica instituída, ou seja, este conhecimento poderá mostrar a contribuição dos cuidados clínicos de enfermagem na saúde da população idosa, dimensionando a prática do trabalho de enfermagem para as reais necessidades dessa clientela.

Os profissionais da saúde devem sempre buscar parcerias com os idosos, seus familiares, o próprio serviço de saúde e toda a sociedade, visando contribuir para a preservação da auto-estima e independência funcional do idoso ${ }^{8}$. Complicações decorrentes de doenças crônicas como a hipertensão, devem ser postergadas ao máximo, para que a dependência e as incapacidades manifestadas pelos idosos sejam somente aquelas decorrentes do próprio envelhecimento, ou seja, passíveis de fácil adaptação e não aquelas que venham a comprometer excessivamente suas atividades de vida diária.

Seguindo este raciocínio, vemos o enfermeiro como peça fundamental no auxílio ao idoso em tratamento farmacológico para HA, procurando minimizar os riscos do surgimento de complicações e outras doenças associadas, evitando o abandono da terapêutica e incentivando o idoso a sentir-se co-responsável por sua saúde.

Com este estudo, pretendemos principalmente chamar a atenção dos enfermeiros para a importância de se conhecer todos os domínios que envolvem o cuidado global ao idoso. Para realizar um cuidado efetivo e com qualidade, o enfermeiro deve buscar aprofundar seu conhecimento sobre os aspectos clínicos, farmacológicos, fisiológicos e psicológicos do envelhecimento, além de ampliar suas técnicas e fundamentos da educação em saúde voltada para esta parcela da população, suas famílias e cuidadores formais ou informais.

Para isso, objetivamos averiguar o risco coronariano ao qual idosos em tratamento anti-hipertensivo estão submetidos, com vistas a subsidiar o cuidado de Enfermagem a esse grupo.

\section{Casuística e métodos}

O estudo é do tipo descritivo, transversal, com natureza quantitativa. Foi realizado junto a um grupo de idosos vinculado à Secretaria de Assistência Social e à Secretaria de Saúde do município de Fortaleza (CE), no período de maio a dezembro de 2007.

O grupo contou com cerca de 150 idosos cadastrados, procedentes de vários bairros de Fortaleza e de outros municípios circunvizinhos à capital. Eles têm duas reuniões semanais, com duração média de duas horas. De acordo com observações prévias, aliadas às informações dadas pela coordenação responsável pelo grupo, verificou-se a freqüência média de 60 idosos participantes por reunião. 
O ingresso na instituição para a coleta dos dados necessários ao andamento da pesquisa foi efetivado com autorização da coordenação da mesma, mediante encaminhamento de ofício, juntamente com o projeto da pesquisa. Já os participantes selecionados para a investigação assinaram o Termo de Consentimento após o esclarecimento dos objetivos do estudo.

A população estudada foi composta por todos os idosos que apresentavam diagnóstico médico de hipertensão arterial, sendo a amostra constituída por aqueles que atenderam os seguintes critérios de inclusão amostral: estar o(a) idoso(a) consciente e orientado(a) durante a coleta dos dados; ter o diagnóstico médico de hipertensão arterial há pelo menos um ano; estar em tratamento medicamentoso para hipertensão arterial há pelo menos seis meses; comparecer a, pelo menos, três reuniões do grupo, durante o período da coleta de dados (devido à necessidade de verificação da pressão arterial em pelo menos três momentos distintos).

Após a análise dos cadastros do grupo foram identificados 62 idosos com diagnóstico prévio de hipertensão e em tratamento farmacológico, sendo que desses, seis não compareceram às reuniões em que a pesquisadora estava colhendo as informações; dois idosos encontravam-se em tratamento medicamentoso há menos de seis meses, contados a partir do início da coleta. Com isso, 54 idosos, de ambos os sexos, em tratamento anti-hipertensivo foram incluídos no estudo.

A coleta de dados ocorreu em agosto e setembro de 2007, no espaço destinado às reuniões do grupo, seguindo as seguintes etapas:

a) Aplicação do formulário de investigação das principais características sócio-demográficas (sexo, faixa etária, estado civil, escolaridade e renda mensal) e clínico-epidemiológicas (valores da pressão arterial, cálculo da adesão terapêutica e investigação de outros fatores de risco para desenvolvimento de doenças coronarianas) no primeiro encontro com o idoso. Salienta-se que o grau de adesão ou não-adesão dos idosos deste estudo foi calculado por meio da escala de avaliação da adesão terapêutica proposto por Moreira’, que abrange aspectos não-farmacológicos e farmacológicos do tratamento da hipertensão.

b) Aplicação da Tabela de Risco Coronariano da American Heart Association $^{10}$ no segundo encontro com o idoso.

c) Verificação da pressão arterial (PA), nos encontros anteriores com o idoso e em mais um encontro, obedecendo-se à técnica de verificação da pressão arterial normatizada pelas V Diretrizes Brasileiras de Hipertensão Arterial ${ }^{11}$. Os valores tensionais foram aferidos utilizando esfigmomanômetro aneróide, previamente calibrado, regulado e atestado pelo Inmetro, obedecendo-se o tamanho do manguito adequado à circunferência do braço do idoso e estetoscópio biauricular de uso pessoal da pesquisadora. Os indivíduos encontravam-se na posição sentada, de acordo com as condições de cada participante. A verificação foi realizada após cinco minutos de repouso de cada participante.

Os dados foram analisados descritivamente e estatisticamente, quando foram calculadas as médias e desvios padrão das variáveis quantitativas. Analisou-se a associação entre variáveis utilizando-se os testes de $\chi^{2}$, razão de verossimilhança e o coeficiente de correlação. Para todos os testes estatísticos, considerou-se como significante quando $\mathrm{p}<0,05$. Os dados foram processados no software SPSS versão 13.0 .

Atendendo às recomendações da Resolução 196/96 do Conselho Nacional de Saúde, referentes às pesquisas envolvendo seres huma$\operatorname{nos}^{12}$, o estudo foi avaliado pelo Comitê de Ética em Pesquisa da Universidade Estadual do Ceará, sendo aprovado para implementação sob o protocolo de $\mathrm{N}^{\circ} 07290341-4$.

\section{Resultados e discussão}

Quando realizado o levantamento das principais características sócio-demográficas do grupo em questão, observou-se que houve a prevalência do sexo feminino (40 idosas) ante ao sexo masculino (14 idosos), equivalente a outros estudos realizados anteriormente pela pesquisadora ${ }^{7,13}$ e a algumas outras pesquisas ${ }^{8,14-15}$. Justifica-se este número à maior participação das mulheres em grupos, não significando maior incidência de HA em mulheres. As mais recentes Diretrizes de Hipertensão Arterial $^{11}$ revelam que, mesmo a prevalência global da HA sendo discretamente maior entre os homens, o sexo não pode ser considerado fator de risco para esta doença.

No que se refere à faixa etária, $26(48,1 \%)$ indivíduos estavam na faixa entre 70 e 79 anos, sendo que, dos 14 homens pesquisados, nove encontravam-se nessa faixa e, entre as idosas, metade encontrava-se prioritariamente entre 60 e 69 anos. A idade máxima dos participantes do estudo foi de 87 anos. Este dado pode ser explicado como resultado do expressivo aumento da expectativa de vida e do envelhecimento populacional, em que as doenças infecciosas e parasitárias cederam lugar às doenças crônico-degenerativas, e como já foi dito, a hipertensão é uma das principais representantes desse tipo de doença. É esperada uma maior participação em grupo de idosos nas faixas etárias mais jovens. Os idosos acima de 80 anos, em sua maioria, têm dificuldades que não permitem este tipo de atividade.

Em relação ao estado civil dos idosos, vimos que metade dos participantes da pesquisa foi de indivíduos casados, uma porcentagem considerável de viúvos entre os idosos (37\%) e sete idosos sem companheiro atual (separados ou solteiros).

Já quanto à escolaridade, 25 idosos apresentaram menos de nove anos de estudo completos (o que representa o Ensino Fundamental) e 18 idosos apenas leram e/ou assinaram o próprio nome, mas não apresentaram escolaridade comprovada. Apenas quatro idosos comprovaram ter mais de nove anos completos de estudo (representando o atual Ensino Médio).

É importante ressaltar que a idade acima de 60 anos já se constitui um fator de risco para o desenvolvimento de complicações relativas à HA, o que torna os idosos pertencentes aos grupos de risco médio ou alto na estratificação do risco cardiovascular ${ }^{11}$. Sabendo disso, a atua- 
ção do enfermeiro é vital na redução da incidência dos fatores de risco modificáveis ou na manutenção de um bom estado de saúde do idoso por ele atendido.

A renda mensal familiar prevalente em mais da metade do grupo foi de um salário mínimo (51,9\% dos idosos), proveniente da aposentadoria ou pensão, sendo que esta quase sempre representava a única fonte de renda de toda uma família.

Os valores da pressão arterial dos idosos foram verificados em três momentos distintos. Vale ressaltar que, para a classificação dos valores tensionais, foram levados em conta os parâmetros diagnósticos das $\mathrm{V}$ Diretrizes Brasileiras de Hipertensão Arterial e, quando as pressões sistólica e diastólica situaram-se em categorias diferentes, o maior valor foi utilizado para classificação da $\mathrm{PA}^{11}$.

$\mathrm{Na}$ primeira verificação, foram encontrados $66,7 \%$ dos idosos com a pressão arterial ótima ou normal (ou seja, controlada) e 11 idosos apresentando hipertensão sistólica isolada. No segundo momento, o número de idosos com a pressão dentro dos padrões normais subiu para 37 e o de idosos com HA sistólica diminuiu para oito. Já na terceira verificação, vimos que o número de idosos com pressão normal voltou a 36 (semelhante à primeira verificação), havendo aumento significativo no número de idosos com os valores limítrofes para a hipertensão (na primeira avaliação, foram quatro idosos e na última, seis idosos). Uma idosa investigada mantevese com hipertensão grave nas três verificações, sendo orientada a respeito de tal situação.

Ao verificar-se a presença da hipertensão nesse grupo (valores tensionais não controlados, mesmo estando os idosos em tratamento), temos que na primeira verificação esse valor foi de $25,9 \%$ e na segunda e terceira verificações, o valor caiu para 22,2\%. Apesar dos dados encontrados apresentarem similaridade com a literatura especializada, reforçamos a importância da atuação constante da enfermagem no controle das cifras pressóricas de seus clientes.

Quando se busca avaliar valores da pressão arterial, temos que ter em mente que o conhecimento dos alvos ideais para controle da PA é importante quando se quer oferecer o melhor prognóstico para os pacientes hipertensos sob tratamento. No caso da população idosa, o valor da pressão a ser obtido por meio do tratamento deve levar em conta a presença de outros fatores de risco cardiovasculares, de doenças concomitantes à HA, de lesões ou danos em órgãos-alvo. As Diretrizes recomendam redução gradual da pressão em idosos para valores abaixo de 140 x $90 \mathrm{mmHg}$, respeitando-se as características individuais e a qualidade de vida dos indivíduos ${ }^{11}$.

É curioso lembrar que até a metade dos anos 80, o tratamento da hipertensão arterial nos idosos era considerado desnecessário, sendo a redução da pressão sistólica isoladamente tida como potencialmente perigosa para os mesmos ${ }^{16}$.

Apesar da hipertensão sistólica isolada (HSI) ser a mais prevalente entre os idosos, estando mais associada a eventos cardiovasculares que a hipertensão diastólica ou a sisto-diastólica ${ }^{17,18}$, encontramos uma mé- dia de nove idosos com hipertensão sistólica isolada, que pode ser considerada baixa. A ocorrência da hipertensão sistólica pode ser explicada pelas alterações anatômicas e fisiológicas das grandes artérias próprias do envelhecimento ${ }^{18}$.

Esses casos de HSI encontrados, além da idosa que apresentou hipertensão grave (estágio III) nos três encontros, foram notificados tanto à coordenação do grupo, quanto aos familiares dos mesmos. Foi solicitada ainda a presença de um profissional médico da Unidade de Saúde localizada próxima ao local dos encontros grupais, para que após uma nova avaliação, fossem prescritos medicamentos anti-hipertensivos de urgência para o controle imediato da PA desta idosa. Além disso, todos os idosos foram orientados a comunicar essas ocorrências aos profissionais de saúde que os acompanhavam.

Alguns autores ressaltam um ponto intrigante relativo ao tratamento anti-hipertensivo, quando se trata de casos de hipertensão sistólica isolada: até que ponto pode-se reduzir a pressão diastólica (PAD) sem causar danos ao indivíduo? Após a metanálise de grandes estudos sobre hipertensão sistêmica, estes mesmos autores concluíram, entre outras coisas, que a PAD de idosos não deve ser inferior a $65 \mathrm{mmHg}$, pois a influência da pressão de pulso (PAS - PAD) também deve ser considerada ao se avaliar o risco cardiovascular ${ }^{19}$.

Quando calculado o risco de desenvolvimento de doença coronariana ao qual os idosos estavam submetidos, considerado de acordo com a tabela de risco coronariano ${ }^{10}$ desenvolvida pela American Heart Association em 1979, foi verificado que os participantes se distribuíram em apenas três categorias das seis listadas pela tabela de risco: sem risco coronariano ( 0 a 8 pontos); risco potencial ( 9 a 17 pontos); risco moderado (18 a 40 pontos); risco coronariano alto (41 a 59 pontos); faixa de perigo (60 a 67 pontos) e perigo máximo (68 pontos).

Foram encontradas apenas duas idosas (4\%) na faixa "sem risco", enquanto que na faixa "risco potencial", tivemos três homens e 19 mulheres (41\%) e no "risco moderado", encontramos 12 homens e 18 mulheres (55\%), com média de 17,65 e desvio padrão de $\pm 6,244$.

Os idosos até 69 anos apresentaram, em sua maioria, risco moderado para desenvolvimento de doenças coronarianas, sendo que para a faixa etária de 70 anos ou mais, os resultados encontrados foram semelhantes. Os homens se apresentaram em uma faixa de risco maior (risco moderado) se comparados às mulheres (sem risco ou risco potencial), comprovado pelo valor de $\mathrm{p}=0,008$.

É sabido que nos homens, a estimativa das taxas de hipertensão costuma ser maior naqueles entre 40 e $50 \operatorname{anos}^{11}$. Quando o indivíduo chega aos 60 anos, caso não tenha adotado nenhuma medida de controle da PA, possivelmente vai apresentar alguma complicação cardiovascular decorrente da doença. Isto ocorre pelo progressivo acúmulo das placas de ateroma, menor eficiência dos mecanismos reguladores da PA e maior freqüência de insuficiência vascular cerebral ${ }^{17}$. 
Entre os indivíduos casados, o risco coronariano encontrado foi moderado, enquanto que para os solteiros, separados e viúvos, a maioria dos indivíduos apresentou risco potencial ou estava sem risco de desenvolvimento de doença coronariana.

As doenças coronarianas são responsáveis por cerca de 300.000 óbitos por ano no Brasil ${ }^{20}$. Por si só, a idade tem sido mostrada como um fator independente para a doença coronariana, mas especificamente no idoso, este risco tende a ser maior, devido à concomitância de doenças, principalmente a hipertensão, o diabetes e as dislipidemias.

Ao aplicar a tabela de risco na população de idosos em tratamento anti-hipertensivo, encontrar quase a totalidade deles em risco de desenvolvimento de doenças coronarianas $(41 \%$ em risco potencial e $55 \%$ em risco moderado) apresenta-se como um dado preocupante e que precisa ser bem avaliado não só pelo enfermeiro, mas por toda a equipe multidisciplinar, já que a doença coronariana é, ainda, causa de cerca de $60 \%$ dos infartos ocorridos em indivíduos com mais de 65 anos, sendo que os sintomas de isquemias miocárdicas nos idosos podem passar despercebidos e serem atribuídos a outras doenças ou simplesmente atribuídos ao processo de senescência, o que aumenta o risco do idoso desenvolver maiores complicações cardiovasculares $^{16}$.

Quando se trata do controle dos fatores de risco avaliados, o papel da enfermagem é fundamental, reforçando a importância dos hábitos de vida saudáveis, estimulando o idoso a aderir ao tratamento não medicamentoso, com vistas a reduzir seus valores pressóricos e seu risco coronariano, já que de todos os fatores, somente os antecedentes familiares e a idade não podem ser modificados.

Outra associação levantada pela pesquisa com os idosos foi a relação entre a adesão terapêutica ao tratamento anti-hipertensivo e o risco coronariano. Após a aplicação da escala de adesão ${ }^{9}$ no grupo de idosos foi identificado que dos 54 em questão, apenas 14 idosos apresentaram adesão ideal (dois homens e 12 mulheres). Dos 40 idosos que apresentaram algum grau de não-adesão, 32 idosos foram classificados na categoria não-adesão leve (nove homens e 23 mulheres) e oito foram incluídos na categoria não-adesão moderada (quatro homens e quatro mulheres).

Uma associação estatisticamente significante entre o risco coronariano e a adesão $(p=0,003)$, além de uma correlação inversamente proporcional $(r=-0,399)$ pode ter ocorrido por conta das características semelhantes no momento da avaliação de cada variável (por exemplo, o sexo, a idade, os hábitos de vida e os valores tensionais). Isso significa que quanto menor a adesão à terapêutica medicamentosa e de modificação do estilo de vida, provavelmente haverá um maior risco de desenvolvimento de doenças coronarianas pelo idoso. Um estudo revelou que um dos maiores motivos de interferência na adesão ao tratamento medicamentoso é o uso de várias medicações concomitantemente, em mais de 30\% dos idosos, seguido da presença de efeitos colaterais intensos, em mais de $20 \%$ dos idosos ${ }^{21}$.
Se o idoso interrompe, modifica por conta própria ou cancela seu tratamento anti-hipertensivo por conta destes ou outros fatores, reduzindo sua adesão, com certeza seu risco coronariano aumentará. A enfermagem deve orientar o idoso a não modificar aleatoriamente o tratamento instituído, mostrando as conseqüências, como o surgimento de outras doenças pela não ingestão de um remédio, pela ingestão de doses erradas ou pela não aceitação de alguma modificação de hábitos, além da chance de mudança no tratamento pela crença do profissional que o remédio ou a ação terapêutica prescrita não está funcionando. Mais uma vez a comunicação da equipe com o portador de hipertensão torna-se instrumento eficaz, influenciando o idoso a discutir a situação com os profissionais na busca de alternativas viáveis para todos?

Houve correlação diretamente proporcional dos valores tensionais com o cálculo do risco coronariano, ou seja, quanto maior o valor das pressões sistólicas, maior o risco de desenvolvimento de doenças coronarianas ao qual o idoso está submetido $(\mathrm{r}=0,316 \mathrm{e} \mathrm{p}=0,020$ na primeira verificação da PAS; $r=0,299$ e $\mathrm{p}=0,028$ na segunda; $\mathrm{r}=$ 0,318 e p $=0,019$ na terceira verificação da PA sistólica).

Por ser uma ferramenta antiga, a tabela de avaliação do risco coronariano da American Heart Association leva em consideração somente o valor da pressão sistólica para seu cálculo. Apesar da hipertensão sistólica possuir alta prevalência entre os idosos e estar fortemente associada a eventos cardiovasculares ${ }^{18}$, cremos que para uma avaliação mais completa do idoso, deve-se levar em consideração os dois valores da PA (sistólica e diastólica).

O conhecimento detalhado sobre cada fator de risco, suas associações e importância podem propiciar a criação de estratégias, visando interferir diretamente nesses fatores. São essas medidas que têm começado a tornar possível a redução da incidência das doenças do coração ${ }^{22}$.

Com isso, conclui-se que apesar de estarem em tratamento farmacológico para hipertensão arterial há algum tempo e em constante acompanhamento multiprofissional de sua saúde, os idosos integrantes do grupo ainda sofrem com problemas relacionados à hipertensão, acarretando no aumento do risco desses indivíduos desenvolverem uma série de complicações decorrentes da doença.

A identificação e o conhecimento dos fatores de risco cardiovasculares em grupos populacionais, como neste caso, os idosos, é fundamental para o desenvolvimento de estratégias de saúde pública na prevenção primária visando a redução da morbi-mortalidade desses problemas de saúde pública. Este é um dos aspectos a serem observados pelo enfermeiro no cuidado a essa população.

Embora o estudo apresente dados interessantes, limitações não podem ser ignoradas. A população investigada tem suas características bem particulares, o que pode tornar inviável a generalização dos resultados para a população geral de hipertensos, a não ser que a comparação desses resultados seja realizada com os de outros grupos de idosos, que apresentem características sócio-demográficas semelhantes. 
Entretanto, todos os dados colocados são altamente dignos de reflexão pelo enfermeiro. Este tema apresentou-se de forma bastante ampla e complexa e ainda há muito o que se pesquisar acerca da temática em questão. Espera-se ter contribuído um pouco mais para as discussões sobre o assunto e para o desenvolvimento do conhecimento existente sobre o cuidado clínico de enfermagem e a hipertensão arterial em idosos.

Por conta de todo o contexto apresentado anteriormente, a questão do idoso portador de hipertensão continua a nos levar à busca de soluções visando contribuir mais no seu tratamento. Como próximo passo, pretende-se desenvolver um projeto maior de intervenção junto aos idosos do grupo pesquisado, visto que a prevenção de complicações associadas à hipertensão arterial poderá ser favorecida pelo enfermeiro ao implementar tecnologias de cuidado sistêmicas, por meio dos diagnósticos, intervenções e resultados de enfermagem, que possibilitem o envolvimento pessoal, interpessoal e social do cliente no seu tratamento, contribuindo para o planejamento e implementação de ações de saúde mais próximas da percepção de tempo e espaço da população, facilitando a eficácia dessas ações.

\section{Agradecimentos}

Parte do projeto foi financiada pela Fundação Cearense de Apoio ao Desenvolvimento Científico e Tecnológico (FUNCAP), por meio de bolsa de formação acadêmica. Os demais gastos com o estudo foram assumidos pela pesquisadora.

\section{Referências}

1. Lueckenotte A. Avaliação da enfermagem para a pessoa idosa. In: Lueckenotte A. Avaliação em gerontologia. $3^{a}$ ed. Rio de Janeiro: Recichmann \& Affonso; 2002. p. 1-3.

2. Coelho Neto A, Araújo ALC. Vida longa com qualidade. Rio-São PauloFortaleza: ABC Editora; 2003. $244 p$

3. Rouquayrol MZ, Almeida Filho N. Epidemiologia e saúde. 6a ed. Rio de Janeiro: Medsi; 2003.

4. Brasil. Ministério da Saúde. Hipertensão arterial sistêmica (HAS) e diabetes mellitus (DM): protocolo. Departamento de atenção básica. Área técnica de diabetes e hipertensão arterial. Brasília: Ministério da Saúde, 2001.

5. Stuchi RA, Carvalho EC. Crenças dos portadores de doença coronariana segundo o referencial de Rokeach sobre o comportamento de fumar. Rev Latino-Am Enfermagem 2003;11(1):74-9.

6. Zaslavsky C, Gus I. Idoso. Doença cardíaca e comorbidades. Arq Bras Cardiol 2002;79(6):635-9.

7. Oliveira CJ, Araujo TL, Moreira TM. Idosos com hipertensão arterial: interferências em sua qualidade de vida. Rev Baiana Enfermagem 2002;17(3):109-12.

8. Silva MJ, Lopes MV, Araújo MF, Moraes GL. Avaliação do grau de dependência nas atividades de vida diária em idosos da cidade de Fortaleza - Ceará. Acta Paul Enferm 2006;19(2):201-6.

9. Moreira TMM. Tecnologia de cuidado na busca da adesão ao tratamento da hipertensão arterial: desenvolvimento e avaliação de uma experiência em Fortaleza-Ceará. Fortaleza, 2003. [Tese de Doutorado]. Ceará: Programa de Pós-Graduação em Enfermagem da Faculdade de Farmácia, Odontologia e Enfermagem da Universidade Federal do Ceará; 2003.

10. Sociedade Brasileira de Cardiologia. Teste o seu coração. Rio de Janeiro. [Acesso em: 03 jul 2007]. Disponível em: http://prevencao.cardiol.br/ testes/.

11. Sociedade Brasileira de Cardiologia, Sociedade Brasileira de Hipertensão, Sociedade Brasileira de Nefrologia. V Diretrizes Brasileiras de Hipertensão Arterial. Rev Bras Hipertens 2006;13(4):260-312.
12. Ministério da Saúde. Conselho Nacional de Saúde. Normas de pesquisa envolvendo seres humanos. Resolução № 196/96. Rev Bioética 1996;4(2):15-25

13. Oliveira CJ, Araujo TL. Plantas medicinais: usos e crenças de idosos portadores de hipertensão arterial. Revista Eletrônica de Enfermagem 2007;9(1):93-105. Disponível em: http://www.fen.ufg.br/revista/v9/v9n1a07.htm

14. Tavares DM, Pereira GA, Iwamoto HH, Miranzzi SS, Rodrigues LR, Machado AR. Incapacidade funcional entre idosos residentes em um município do interior de Minas Gerais. Texto Contexto Enferm 2007;16(1):32-9.

15. Menéndez J, Guevara A, Arcia N, Díaz EM, Marín C, Alfonso JC. Enfermedades crónicas y limitación funcional en adultos mayores: estudio comparativo en siete ciudades de América Latina y el Caribe. Rev Panam Salud Publica 2005;17(5/6):353-61.

16. Sociedade de Cardiologia do Estado do Rio de Janeiro. Recomendações da SOCERJ: manejo terapêutico em cardiogeriatria. Rev SOCERJ 2004;17/Supl B):7-96

17. Grupo de Estudos em Cardiogeriatria da Sociedade Brasileira de Cardiogeriatria. I Diretrizes do Grupo de Estudos em Cardiogeriatria. Arq Bras Cardiol 2002;79(Supl I):4-46.

18. Liberman A. Aspectos epidemiológicos e o impacto clínico da hipertensão no indivíduo idoso. Rev Bras Hipertens 2007;14(1):17-20.

19. Freitas EV, Brandão AA, Magalhães ME, Pozzan R, Brandão AP. Qual a pressão arterial diastólica aceitável no tratamento de hipertensão sistólica isolada? Rev Bras Hipertens 2007;14(1):25-8.

20. Gus I, Fischmann A, Medina C. Prevalência dos fatores de risco da doença arterial coronariana no estado do Rio Grande do Sul. Arq Bras Cardiol 2002;78(5):478-83.

21. Blanski CR, Lenardt MH. A compreensão da terapêutica medicamentosa pelo idoso. Rev Gaúcha Enferm. 2005;26(2):180-8.

22. Lélis Filho R. Identificação do perfil de risco cardiovascular em trabalhadores de uma indústria automobilística: aplicação da tabela da Sociedade Americana de Cardiologia. [Dissertação de Mestrado]. Rio de Janeiro: Universidade Federal Fluminense; 2006. 96 p. 